\title{
A Comparison Of Mean-Variance And Mean-Semivariance Optimisation On The JSE
}

\author{
Jiten Vasant, University of Cape Town, South Africa \\ Laurent Irgolic, University of Cape Town, South Africa \\ Kanshukan Rajaratnam, University of Cape Town, South Africa \\ Ryan Kruger, University of Cape Town, South Africa
}

\begin{abstract}
This study investigates the effectiveness of semivariance versus mean-variance optimisation on a risk-adjusted basis on the JSE. We compare semivariance and mean-variance optimisation prior to, during and after the recent financial crisis period. Additionally, we investigate the inclusion of a fixed-income asset in the optimal portfolio. The results suggest that semivariance optimisation on the JSE in a pure equity case produces lower absolute returns, yet superior risk-adjusted returns. Further investigation suggests that semivariance metrics are effective within a certain range of portfolio sizes and diminishes in benefit once portfolios become larger. A fixed income asset scenario tested under the hypothesis of semivariance optimisation favoured greater bond weightings in optimal portfolios.
\end{abstract}

Keywords: Semivariance; Johannesburg Stock Exchange, Optimisation, Mean-Variance

\section{INTRODUCTION}

Portfolio theory has been evolving ever since its inception by Harry Markowitz and his pioneering research shaped the portfolio risk-return model known as mean-variance optimisation. A newer concept of post-modern portfolio theory is that of downside risk metrics for risk measurement in the context of portfolio optimisation. Previously the convention was to use a mean-variance strategy to determine the efficient frontier for a portfolio. The foundations of this theory however, are based on a set of strict assumptions with the result that the majority of models fail to capture reality perfectly and exhibit significant model risk. Downside risk optimisation, which models the efficient frontier using semivariance, has exhibited potential for providing better risk metrics.

This paper aims to test the use of semivariance as a more realistic method of portfolio optimisation in a South African context. While empirical testing has been investigated on foreign markets, studies relating to South Africa in particular are limited. Furthermore, the use of downside risk optimisation has implications for the management of pension funds, thus providing cause for research.

In addition, portfolio size will be examined in relation to both optimisation models. Prior research in other financial markets suggests that larger-sized portfolios are indifferent to the method of optimisation used. The effects of including a fixed-income asset will also be tested against both models.

The remainder of this paper is structured as follows. Section 2 reviews prior literature on the development of mean-variance and semivariance. Section 3 presents our data and discusses the methodology adopted in testing the hypotheses. The results are presented in Section 4 while Section 5 concludes. 


\section{LITERATURE REVIEW}

Harry Markowitz pioneered the research into determining optimal portfolios in 1952. Markowitz (1959) concluded that the optimal portfolio is one that provides the best combination of risk and return for a specific investor, depending on said investor's degree of risk tolerance. As a result, Markowitz introduced the concepts of variance and standard deviation with regards to the calculation of portfolio risk. Markowitz (1959) also identified two objectives of the common investor; high returns are preferred to low returns and stability in returns is preferred to volatility. These conclusions lead to the formation of efficient portfolios (points representing risk versus return on a Cartesian plane) and introduced the efficient frontier (a curve that provides the optimal set of efficient portfolios).

Subsequently, Tobin (1958formulated the liquidity preference theory. In addition to Markowitz's efficient frontier, Tobin accounted for the risk preferences of investors via indifference curves. Tobin (1958) states that an investor is assumed to have preferences between expected return and risk that can be represented by a field of indifference curves. It is these theories that formed the core of mean-variance strategies for portfolio optimisation used today.

These theories do, however, employ a number of assumptions that dissociate their results from real world outcomes. Markowitz $(1952,1959)$ assumes that asset returns are fully explained under a normal (symmetrical) distribution by the distribution's first two moments i.e. mean and variance. Higher order moments (skewness and kurtosis) were thus not required. Leland (1999) states that the distribution of asset returns are generally not normal and investors have significantly different views pertaining to upside and downside risk. The degree to which asset returns are non-normal was investigated by Cont (2001), who suggests that asset returns display excess kurtosis; that is, they exhibit higher peaks and 'fatter' tails than a normal distribution. This has an effect on the risk metrics of variance and standard deviation. The steeper peaks suggest that asset returns are less deviant about their means, while the 'fatter' tails are indicative of the fact that the majority of returns lie further away from the mean. This implies higher occurrences of extreme positive and negative asset returns. Cont (2001) also observed that there is empirical evidence that asset returns have a tendency to return below-mean returns rather than above-mean returns, a statistical phenomenon referred to as skewness. Empirical testing has observed that asset returns tend to demonstrate negative skewness in general. As a result, mean-variance optimisation, while theoretically sound, may not account fully for the empirically-observed behaviour of return distributions. A more accurate model would have to include higher order moments such as skewness and kurtosis to compensate for observed asset returns and investor preferences.

A superior risk metric was proposed in the form of semivariance. The concept of semivariance optimisation can be quantified simply as the fact that investors are more concerned by the underperformance of a portfolio rather than over-performance. In other words investors do not treat risk symmetrically and are more vigilant against downside risk. Ferguson and Rom (1994) established that conventional portfolio theory is justifiably a unique case of post-modern theory under symmetric conditions. Further, and more importantly, they discuss the implication of a target level of return, stemming from the early works on semivariance by Markowitz (1959) and Hogan and Warren (1972). They stipulate that variances are indicative of the risk of achieving average returns, whereas a more modern approach tailors risk specifically to an investor's target return level such that returns above this target return level do not constitute risk. These notions support the prior discussions on the asymmetry of asset return distributions.

While the early work of Markowitz (1959) and Roy (1952) proposed methods for determining semivariance risk metrics, both these methods required time consuming iterative techniques to calculate cosemivariance, as suggested by Cumova and Nawrocki (2011).

Markowitz (1959) proclaimed that analyses based on semivariance produced more accurate portfolios than those based on variance. According to Markowitz (1959) the reason for the popularity of mean-variance optimisation is a direct consequence of its cost effectiveness, computational simplicity and familiarity, whereas semivariance required complex computation algorithms, not feasible with the level of computing power at that time. However, with the drastic improvement of computing power today, efficient calculation of semivariance portfolios is certainly possible. 
However, there is still an inherent complexity in calculating semivariance, which arises from the determination of the co-semivariance matrix. Cumova \& Nawrocki (2011) suggest that the co-semivariance matrix is endogenous versus the exogenous matrix which is generated by normal mean-variance optimisation. Estrada (2008) suggested that the reason for this endogenous behaviour is due the fact that changing the portfolio weights changes the periods in which the portfolio underperforms the benchmark, which in turn will change the cosemivariance matrix. To overcome this issue of an endogenous matrix problem, Cumova \& Nawrocki (2011) suggested two approaches, the first being a heuristic approach where inter-correlations between securities were ignored. The second involves converting the asymmetric endogenous matrix to a symmetric positive semi-definite matrix.

In essence Estrada (2007) presents a solution of co-semivariance based on determining for which periods assets jointly underperform a target return level. The main criticism stems from the fact that both assets need to underperform the target level of return in the same period for the co-semivariance to be valid. Cumova \& Nawrocki (2011) further elaborate on the fact that due to the heuristic nature of the method proposed by Estrada (2007), the results of his method differ. They offer a possible explanation for this, suggesting that Estrada's method employs a smaller number of below-target observations.

The differences in methodologies regarding the co-semivariance matrix have become a prominent topic of debate. Despite differing opinions the general consensus is that not only do semivariance techniques portray a more realistic representation of investors preferences regarding stock returns, but also generate portfolios with superior risk-adjusted returns. However, it is worth noting that empirical testing from which these conclusions have been drawn still suffer from teething problems in circumventing the computational complexity surrounding semivariance.

It is now prudent to investigate the assumption of normality of asset returns specific to the JSE. Mangani (2007) built on the previous work of Page (1993) who concluded that JSE returns are not normally distributed. Mangani (2007) stated that “... there was unequivocal evidence of leptokurtosis on the JSE, a feature that is documented for most markets, and renders no support for the assumption of normality in the distribution of security returns." Furthermore Mangani (2007 concluded that not only did the JSE exhibit leptokurtosis but returns were also negatively skewed.

Since the JSE seems to display the characteristics synonymous with most other markets, empirical testing of semivariance should yield similar results compared to empirical studies on other markets. While semivariance seems to offer superior risk-adjusted returns in all types of portfolios, portfolios that are specifically mandated to behave in a risk-averse manner are of particular importance. One such of these portfolios is pension funds. The implied benefit of semivariance relates to applications in pension funds and could thus improve the performance of these funds. Pension funds are governed by a set of strict regulations, limiting the amount of risk the fund may assume.

Pension funds serve as way employees and individuals can save for their retirement. Large amounts of pooled capital are invested in a number of financial instruments to yield a safe return on the invested funds. Such funds have a great amount of responsibility and hence the Pension Fund Act of 1956 was established to regulate pension fund investments. The Pension Fund Act has since been amended, by Regulation 28, thus allowing more pension fund capital to be invested in alternative investments and yield greater returns. Thus with pension funds being able to assume greater risk, a more vigilant watch over downside risk seems necessary.

\section{DATA AND METHODOLOGY}

\subsection{Data}

Both monthly and daily data was obtained from Thompson Reuters Datastream for a ten-year period spanning 2003 to 2013. Due to the deviations from average historical stock returns caused by the 2008 financial crisis, a control time period was selected which focused on returns from 1996 to 2006. 
Companies constituting the JSE's Top 40 index were considered for the primary model tests since the top 40 shares constitute a large majority of the JSE's capitalization and are highly liquid. For secondary hypotheses testing, a wider array of 140 stocks, which were listed over the full period, was used as a database.

A secondary model made use of the daily returns of the GOVI bond index (an index that tracks the returns on government bonds). The daily returns retrieved spanned from 1998 to 2006 and the daily stock returns were therefore adjusted to match the time period for this particular model.

\subsection{Methodology}

The methodology employed relies heavily on the experiments previously conducted by Estrada (2007, 2008) and Cumova and Nawrocki (2011). Cumova and Nawrocki (2011) hypothesised that the effects of portfolio size on differences between mean-variance and semivariance techniques were significant. Efficient frontiers for both measures were created for portfolios ranging in size from 25 to 150 stocks. The findings suggested that at portfolio sizes greater than 50 stocks, the efficacy of semivariance metrics diminished, in terms of producing superior risk adjusted returns. However, for portfolios below 50 stocks, semivariance metrics tended to outperform. This result is certainly specific to the financial market used in their study. A possible reason for this could be the effects of concentration and diversification, which are inherent in all financial markets. Therefore since the JSE displays characteristics of high concentration, testing the effect of portfolio size on optimisation method was deemed relevant.

The benchmark return value, used to define negative and positive returns in terms of semivariance, was selected as zero. This suggests that any negative return will be viewed as unfavourable and included in the calculation of the semivariance risk metric. The use of a zero return benchmark provides easier and more accurate comparability. A more realistic scenario would employ the average rate of inflation over the time period as the return benchmark; however this would complicate comparisons of different time periods.

The semivariance equation employed also influences the validity of the results. To understand the method of calculating semivariance, it is important to state two equations developed by Markowitz $(1952,1959)$ under mean-variance optimisation:

$E\left(R_{p}\right)=\sum_{i=1}^{n} W_{i} \times R_{i}$
$\sigma_{p}^{2}=\sum_{i=1}^{n} \sum_{j=1}^{n} W_{i} \times W_{j} \times \sigma_{i j}$

Equation (1) relates equity member returns to expected portfolio returns ( $E(R p)$ ), where $R_{i}$ is the individual asset returns $i$ and $W_{i}$ the weighting of asset $i$ in the portfolio. Equation (2) is the variance of the portfolio with $\mathrm{W}_{\mathrm{i}}$ and $\mathrm{W}_{\mathrm{j}}$ reflecting the respective weightings of assets $\mathrm{i}$ and $\mathrm{j}$, and $\sigma_{i j}$ represents the covariances between the two assets $\mathrm{i}$ and $\mathrm{j}$. The above two equations model the conclusions reached by Markowitz and may be used to calculate efficient portfolios that plot the efficient portfolio space. However, Markowitz (1959:196) also suggested the following equations for calculating semivariance:

$\varphi_{p}^{2}=\sum_{i=1}^{n} \sum_{j=1}^{n} W_{i} \times W_{j} \times \varphi_{i j}$

$\varphi_{i j}=\frac{1}{n} \times \sum_{k=1}^{K}\left(R_{i k}-B\right)\left(R_{j k}-B\right)$

Equation (3) is defined as the portfolio's semivariance. Equation (4) calculates the co-semivariance. B represents a target return while $R_{i k}$ and $R_{j k}$ represent the returns of assets $i$ \& $j$ respectively in period $k$, when these returns are less than the benchmark B. Finally $\mathrm{K}$ represents the number of periods for which the portfolio underperforms the benchmark. 
In an earlier paper, Nawrocki (1991) suggested calculating a symmetric co-semivariance matrix as follows:

$\varphi_{i j}=\varphi_{i} \varphi_{j} \rho_{i j}$

Equation (5) represents the symmetric co-semivariance matrix. $\varphi_{i}$ and $\varphi_{j}$ represent the respective semideviations (square root of semivariances) and $\rho_{\mathrm{ij}}$ the correlation between assets. Alternative research conducted by Javier Estrada (2007) yielded a different method for calculating a symmetric co-semivariance matrix. Estrada (2007) suggested the following formula:

$\varphi_{\mathrm{ij}}=\frac{1}{\mathrm{~K}} \times \sum_{\mathrm{k}=1}^{\mathrm{K}}\left[\operatorname{Min}\left(\mathrm{R}_{\mathrm{ik}}-\mathrm{B}, 0\right) \times \operatorname{Min}\left(\mathrm{R}_{\mathrm{jk}}-\mathrm{B}, 0\right)\right]$

For computational ease, equations (3) and (6), outlined by Estrada (2008), was used to calculate the semivariance and co-semivariance matrix of returns respectively. It must be noted that while equation (6) does provide computational ease, it sacrifices validity insofar as that only assets that share negative returns in the same period are included by the equation thus the equation does not capture the complete degree to which stocks vary together when only negative returns are considered.

Portfolio returns, standard deviations and semi deviations were calculated through normal optimisation techniques. The vector $\mu$ represents average returns of assets in the portfolio, $\sigma$ the variance-covariance matrix of returns and the vector $\omega$ the weight of each asset in the portfolio. Therefore:

Portfolio return $=\omega \mathrm{T} \times \mu$

Portfolio variance $=\omega \mathrm{T} \times \sigma \times \omega$

20000 random portfolio weights were generated allowing for 20000 associated portfolio returns, standard deviations and semi deviations to be calculated as per equations (7) and (8). Furthermore two risk-adjusted return (RAR) metrics were calculated for each of the 20000 weightings by dividing the portfolio return by either portfolio standard deviation or portfolio semi deviation.

The following models were constructed to test various hypotheses:

1. A 5 stock and 15 stock portfolio based on daily JSE top 40 data from 1996 to 2006

2. A 5 stock and 15 stock portfolio based on daily JSE top 40 data from 1998 to 2006 with the inclusion of a fixed income asset in each portfolio based on the GOVI index data from 1998 to 2006

3. A 5 stock and 15 stock portfolio based on monthly JSE data from June 1996 to July 2006 (pre-crisis portfolio) and from June 2003 to July 2013 (constituting the financial crisis period and after)

Model one represents the primary test, an impartial comparison of the optimisation method. Model two investigates the addition of a fixed-income asset. Model three compares optimisation methods with respect to postcrisis and financial crisis periods.

The first issue dealt with in terms of portfolio construction in each of the models was the random sampling of stocks upon which portfolios would be generated. This process involved randomly selecting a specific number of stocks from a larger database to create a portfolio, with each portfolio selection being independent from the previous one. Random sampling of stocks versus actively selecting a specific portfolio of stocks serves to reduce any observational bias as well as negate the ability that some stocks may potentially distort results.

Each model and associated hypothesis was tested by creating a loop function. The minimum deviation and semi deviation portfolio (out of the 20000 generated portfolios) as well as corresponding portfolio return was found. Next the risk-adjusted metrics were calculated. This process was repeated five times for five independent random portfolios. The average risk-adjusted metrics were calculated by averaging the aggregated metrics i.e. each average risk-adjusted metric represented the average of five independent random portfolios. This process was then repeated 
fifty times rendering two sets of fifty 5-portfolio average risk-adjusted metrics, one for standard deviation and one for semi deviation.

After running the loop function for each model, the resultant data allows for statistical testing. The general concept is to first determine if there is a difference in the risk-adjusted return metrics produced by both optimisation methods and secondly to statistically test the differences. Two tests were used to analyse the output data, an F-test, to test whether the output had equal variances or not. Second, a t-test was used to decide if at a specific significance level, the average of the output data differed or not.

The biggest constraining element to the analysis was availability of computing power. Ideally 1000 iterations of the loop function would provide more statistically smooth output; however, limitations with computer processing capabilities subvert this. Portfolio sizes are also constrained by computing ability. Larger portfolio sizes would provide more information on the behaviour of semivariance techniques but require more processing power. Lastly, the scope of this analysis is restricted to stocks and a single bond asset, where in reality portfolios comprise several classes of assets.

\section{RESULTS}

The findings of the analysis support the hypothesis that semivariance optimisation provides a better riskadjusted return over traditional mean-variance optimisation for stocks on the JSE.

Table 1: 5 Stock Scenario

\begin{tabular}{|l|r|r|}
\hline t-Test: Paired Two Sample for Means & \\
\hline & RAR_std & RAR_semi \\
\hline Mean & 0.057565 & 0.072327 \\
\hline Variance & $1.73 E-05$ & $3.97 \mathrm{E}-05$ \\
\hline Observations & 50 & 50 \\
\hline Pearson Correlation & 0.912594 & \\
\hline Hypothesized Mean Difference & 0 & \\
\hline df & 49 & \\
\hline t Stat & -34.5359 & \\
\hline$P(T<=t)$ one-tail & $2.33 E-36$ & \\
\hline t Critical one-tail & 1.676551 & \\
\hline$P(T<=t)$ two-tail & $4.67 \mathrm{E}-36$ & \\
\hline t Critical two-tail & 2.009575 & \\
\hline
\end{tabular}


Table 2: 15 Stock Scenario

\begin{tabular}{|l|r|r|}
\hline t-Test: Paired Two Sample for Means & \\
\hline & RAR_std & RAR_semi \\
\hline Mean & 0.071152 & 0.081476 \\
\hline Variance & $1.39 E-05$ & $2.47 \mathrm{E}-05$ \\
\hline Observations & 50 & 50 \\
\hline Pearson Correlation & 0.689169 & \\
\hline Hypothesized Mean Difference & 0 & \\
\hline df & 49 & \\
\hline$t$ Stat & -20.1885 & \\
\hline$P(T<=t)$ one-tail & $1.07 \mathrm{E}-25$ & \\
\hline t Critical one-tail & 1.676551 & \\
\hline$P(T<=t)$ two-tail & $2.14 \mathrm{E}-25$ & \\
\hline$t$ Critical two-tail & 2.009575 & \\
\hline
\end{tabular}

As expected, the average risk-adjusted return (RAR) is significantly greater under semivariance optimisation verses mean-variance (see Table 1 and 2 respectively). However, the average returns associated with these risk-adjusted returns remain higher for both scenarios under mean-variance optimisation. This outcome suggests that while mean-variance techniques generate higher returns, semivariance techniques generate significantly better risk-adjusted returns.

Table 3: Difference In Portfolio Sizes

\begin{tabular}{|l|r|r|}
\hline \multicolumn{3}{|l|}{ t-Test: Two-Sample Assuming Unequal Variances } \\
\hline & & \\
\hline & diff_5 & diff_15 \\
\hline Mean & 0.014762 & 0.010324 \\
\hline Variance & $9.14 \mathrm{E}-06$ & $1.31 \mathrm{E}-05$ \\
\hline Observations & 50 & 50 \\
\hline Hypothesized Mean Difference & 0 & \\
\hline df & 95 & \\
\hline t Stat & 6.658715 & \\
\hline $\mathrm{P}(\mathrm{T}<=\mathrm{t})$ one-tail & $8.89 \mathrm{E}-10$ & \\
\hline $\mathrm{t}$ Critical one-tail & 1.661052 & \\
\hline $\mathrm{P}(\mathrm{T}<=\mathrm{t})$ two-tail & $1.78 \mathrm{E}-09$ & \\
\hline$t$ Critical two-tail & 1.985251 & \\
\hline
\end{tabular}

Another question to deliberate is the efficacy of semivariance as portfolio size increases. Prior research would suggest that semivariance efficacy depreciates as portfolio size increases. The accompanying results in Table 3 , testing the difference in average risk-adjusted return attributable to mean-variance and semivariance optimisation for the 5 and 15 stock scenarios, confirms this hypothesis. The resultant t-test confirms that the efficacy of the semivariance optimisation to return superior risk-adjusted returns has decreased from the 5 stock portfolios (mean difference of 0.014762 ) to the 15 stock portfolios (mean difference of 0.010324). In other words the benefit or ' $g a p$ between methods' achieved by semivariance optimisation has diminished. 
Table 4: 5 Stock And Bond Portfolio

\begin{tabular}{|l|r|r|}
\hline \multicolumn{2}{|l|}{$t$-Test: Paired Two Sample for Means } & \\
\hline & & \\
\hline Mean & std & semi \\
\hline Variance & 0.673482 & 0.681648 \\
\hline Observations & 0.003684 & 0.003956 \\
\hline Pearson Correlation & 50 & 50 \\
\hline Hypothesized Mean Difference & 0.840402 & \\
\hline df & 0 & \\
\hline t Stat & 49 & \\
\hline$P(T<=t)$ one-tail & -1.65078 & \\
\hline$t$ Critical one-tail & 0.052589 & \\
\hline$P(T<=t)$ two-tail & 1.676551 & \\
\hline$t$ Critical two-tail & 0.105179 & \\
\hline
\end{tabular}

Table 5: 15 Stock And Bond Portfolio

\begin{tabular}{|l|r|r|}
\hline \multicolumn{3}{|l|}{ t-Test: Paired Two Sample for Means } \\
\hline & & \\
\hline & \multicolumn{1}{|c|}{ std } & semi \\
\hline Mean & 0.158125 & 0.159159 \\
\hline Variance & 0.000515 & 0.000688 \\
\hline Observations & 50 & 50 \\
\hline Pearson Correlation & 0.233305 & \\
\hline Hypothesized Mean Difference & 0 & \\
\hline df & 49 & \\
\hline t Stat & -0.24035 & \\
\hline P(T<=t) one-tail & 0.405531 & \\
\hline t Critical one-tail & 1.676551 & \\
\hline P ( T<=t) two-tail & 0.811063 & \\
\hline$t$ Critical two-tail & 2.009575 & \\
\hline
\end{tabular}

The next scenario dealt with the inclusion of a bond asset to both the 5 and 15 stock portfolios (see results in Table 4 and 5). It was hypothesised that while minimising deviation as an optimisation technique, bonds would be heavily weighted in the portfolio and furthermore that semivariance techniques would tend to place higher weightings on the bond asset over mean-variance techniques. In Table 4 and 5, std and semi refer to the weighting of the bond asset under each optimisation technique respectively. While the results do not suggest much support for the hypothesis, particularly in the 15 stock cases (average bond weighting of 15.8\% under mean-variance and $15.9 \%$ under semivariance), they do show on average that bonds are weighted higher under semivariance though this is not statistically significant in the 15 stock scenarios. However, with a p-value of approximately 0.053 , the 5 stock scenarios offer encouraging results as the statistical significance is marginal (average bond weighting of 67.3\% under mean-variance and $68.1 \%$ under semivariance). The difference in results may be attributable to the effects of diversification. However, other extraneous variables may be distorting the results like the bond asset chosen or the limited iterations of data recorded. 
Table 6: Pre-Crisis And Crisis Comparison

\begin{tabular}{|l|r|r|}
\hline \multicolumn{2}{|l|}{ t-Test: Two-Sample Assuming Equal Variances } \\
\hline & \multicolumn{1}{|c|}{ Pre diff } & Post diff \\
\hline Mean & 0.031150924 & 0.044531681 \\
\hline Variance & 0.005487686 & 0.008990516 \\
\hline Observations & 50 & 50 \\
\hline Pooled Variance & 0.007239101 & \\
\hline Hypothesized Mean Difference & 0 & \\
\hline df & 98 & \\
\hline$t$ Stat & -0.786336394 & \\
\hline$P(T<=t)$ one-tail & 0.216783869 & \\
\hline$t$ Critical one-tail & 1.660551217 & \\
\hline$P(T<=t)$ two-tail & 0.433567738 & \\
\hline$t$ Critical two-tail & 1.984467455 & \\
\hline
\end{tabular}

The last scenario tested both methods under pre-crisis and crisis periods. This analysis is of particular interest as since the financial crisis, emphasis on downside risk has been greatly increased. The hypothesis suggests that semivariance methods served to outperform mean-variance methods during the crisis. The results in Table 6 summarises the difference in optimisation techniques, where Pre diff relates to the difference in risk adjusted returns pre-crisis (semideviation RAR - standard deviation RAR) and Post diff the difference during and post-crisis. Even though the output is inconclusive as we fail to reject the null hypothesis that the differences between the periods are not statistically significant, the mean difference is still bigger post crisis. A reason as to why this test does not support our hypothesis could be due to the fact that South African stocks on the JSE were well shielded against the more devastating effects of the financial crisis.

\section{CONCLUSION}

The resultant output from conducting the above testing suggests that semivariance optimisation does offer a significant benefit in terms of risk adjusted returns. While traditional methods may still offer higher absolute returns, there is a greater amount of relative risk attached to generating these returns. This supports the hypothesis that riskaverse investors should consider a semivariance approach when constructing portfolios. The efficient space created further supports an investors risk preference in tailoring portfolios based on a downside risk framework. This analysis tested on an empirical level, the feasibility of semivariance on the JSE stock database, with particular emphasis on the top 40. The results show a noticeable improvement in risk adjusted returns for pure stock portfolios in this regard. Adding to the core thesis statement, both fixed income assets and pre-crisis and crisis analysis, showed encouraging results that risk averse investors should weight portfolios more heavily with regard to fixed income assets, and that during periods of recession, semivariance optimisation minimised negative portfolio returns. However, further testing need be employed, to determine the significant result of these two secondary hypotheses.

The scope of this analysis was specific to the JSE top 40. There is opportunity to be expanded into additional equity markets. Firstly, there is an opportunity to expand the portfolio sizes analysed with greater processing power. An important avenue for future research is to test and compare other downside risk metrics to semivariance. Semivariance is a special case of a general measure called the lower partial moment. Other lower partial moments quantify risk differently, thus may prove more applicable.

\section{AUTHOR INFORMATION}

Jiten Vasant is a graduate specialising in Finance and Economics from the University of Cape Town, South Africa

Laurent Irgolic is a graduate of Finance from the University of Cape Town, South Africa. 
Ryan Kruger is a Senior Lecturer of Finance and is an associate and founding member of the African Collaboration for Quantitative Finance and Risk Research (ACQuFRR) at the University of Cape Town, South Africa. His research interests lie in the predictive modelling of asset returns and the application of machine learning in asset pricing.

Email: ryan.kruger@uct.ac.za

Kanshukan Rajaratnam is a Senior Lecturer of Finance and is an associate of the African Collaboration for Quantitative Finance and Risk Research (ACQuFRR) at the University of Cape Town, South Africa. His research interests lie in decision making in consumer credit loans, modelling credit union behaviour, and the Basel Accord. He is a member of the Operations Research Society of South Africa (ORSSA).

Email: kanshukan.rajaratnam@uct.ac.za (corresponding author).

\section{REFERENCES}

1. Cont, R. (2001). Empirical properties of asset returns: stylized facts and statistical issue. Quantitative Finance. 1(2): 223-236.

2. Cumova, D. \& Nawrocki, D. (2011). A symmetric LPM model for heuristic mean-semivariance analysis. Journal of Economics and Business. 63(3): 217-236.

3. Estrada, J. (2007). Mean-semivariance behaviour: Downside risk and capital asset pricing. International Review of Economics and Finance. 16: 169-185.

4. Estrada, J. (2008). Mean-semivariance optimisation: A heuristic approach. Journal of Applied Finance. 18(1): 57-72.

5. Ferguson, K. \& Rom, B. (1993). Post-modern portfolio theory comes of age. The Journal of Investing. 2(4): 27-33.

6. Firer, C. Neu-Ner, M A. (1997). The benefits of diversification on the JSE. Investment Analysts Journal. 46: 45-59.

7. Galagedera, D. (2007). A review of capital asset pricing models. Managerial Finance. 33(10): 821-832.

8. Hogan, W. \& Warren, J. (1972). Computation of the Efficient Boundary in the E-S Portfolio Selection Model. The Journal of Financial and Quantitative Analysis. 7(4): 1881-1896.

9. Huelin, L. \& Mirza, K. (2011). Portfolio optimisation n a downside risk framework. Germany: Lap Lambert Academic Publishing.

10. Leland, H E. (1999). Beyond Mean-Variance: Performance Measurement in a Nonsymmetrical World. Financial Analyst Journal. January/February: 29-36.

11. Lintner, J. (1965). The Valuation of Risk Assets and the Selection of Risky Investments in Stock Portfolios and Capital Budgets. The Review of Economics and Statistics. 47(1): 13-37.

12. Mangani, R. (2007). Distributional properties of JSE prices and returns. Investment Analysts Journal. 66: 57-72.

13. Markowitz, H M. (1959). Portfolio Selection: efficient diversification of investments. New York: John Wiley \& Sons Inc.

14. Morse, D. (1984). An Econometric Analysis of the Choice of Daily Versus Monthly Returns in Tests of Information Content. Journal of Accounting Research. 22(2): 605-623.

15. Roy, A. (1952). Safety first and the holding of assets. Econometrica. 20(3): 431-449.

16. Sharpe, W F. (1964). Capital Asset Prices: A Theory of Market Equilibrium under Conditions of Risk. The Journal of Finance. 19(3): 425-442.

17. Tobin J. (1958). Liquidity Preference as Behavior Towards Risk. The Review of Economic Studies. 25(2): 65-86.

18. Van Rensburg, P. (2001). A Decomposition of Style-Based Risk on the JSE. Investment Analysts Journal. 54: 45-60. 